\title{
Prediction of Transient Stability Using Wide Area Measurements Based on ANN
}

\author{
Mohammad M. Al-Momani ${ }^{1}$, Seba F. Al-Gharaibeh ${ }^{2}$ \\ ${ }^{1}$ Protection Engineer, Jordan, monqedmohammad@gmail.com \\ ${ }^{2}$ Research Scholar, Jordan, 620180441015@ muath.edu.jo
}

Received Date : October 02, 2021 Accepted Date : October 24, 2021 Published Date : November 07, 2021

\begin{abstract}
The concept of wide-area control and protection as an application on real-time wide-area measurement systems makes the transient stability prediction more accurate in early time after fault occurrences. The transient prediction is the first step in the dynamic control system to avoid any unwanted emergency or non-stable power system state. In this paper, an early predictionof the power system stability once the fault cleaning using real-time dynamic data collected by WAMS is proposed based on an artificial neural network (ANN). The dataset collected by the different contingency analyses on the IEEE 39 bus test system is used to train a multilayer perceptron network. Pre-fault, duringfault, and post-fault generators' speeds are fed to ANN as inputs, and the status of the overall system, either stable or not, is the output of ANN. The proposed model can predict an unstable state within $100 \mathrm{~ms}$ after the fault. NEPLAN simulator is used to simulate the dynamic analysis ofthe IEEE 39-Bus test system, and MATLAB 2019a is used to design the ANN.
\end{abstract}

Key words: wide-area monitoring system (WAMS), phasor measuring unit PMU, transient stability, wide-area control system, ANN, NEPLAN, MATLAB.

\section{INTRODUCTION}

Electric power systems should supply a continuous and secure service for the consumers while considering the economic dispatch. However, different types of faults endanger their stability and, in the worst case, causing a loss of stability, leading to a blackout of the power system [1-2]. Wide-area protection and control (WAPAC) systems are proposed to prevent the power system loss of stability after critical events [3-4]. WAPAC systems use the phasor measurement units (PMU) information to counteract the propagation of severe faults [5]. Predicting the power system's post-fault transient stability afteran event is significant for preventing any potential loss of stability. This prediction increases the chance for the WAPAC systems to trigger corrective control actions as fast as possible against the potential instability, and hence, the dynamic security of the power system is enhanced.
If an unstable case is predicted early, the prevention system from unwanted scenarios may be more applicable. The WAMS plays a vital role in sensing, collecting, and processing real-time power systems dynamically based on GPS technology. Table 1: Comparative Analysis

The problem of power system transient instability prediction after fault clearance is addressed in many articles. Based on the literature, the problem may be categorized into (a) timedomain simulations [6-7]. (b) transient-energy-function (TEF) methods [8-9] and (c) machine-learning techniques [10]. The time-domain simulation method is a dynamic behavior of the power system, so a huge set of differential equations need to solve. TEF method uses the Lyapunov stability principle to determine the dynamic status of the power system without using the complex differential equations sets. ANN is a common example of machinelearning techniques. All these techniques need to know the operational point before the fault and the post fault status. However, the power system's stability depends not only on the post and pre-fault system operation but also on the fault location, duration, and characteristic.

In [11], an artificial neural networks-based methodology is proposed for directly predicting power system stability after clearing the fault. A dataset is generated to train a multilayer perceptron offline; then, it is used for early online prediction of any transient instability. The inputs feed the neural network: pre-fault, during-fault, and post-fault voltage magnitude measurements collected from the phasor measurement units. This study depends on the fault location and duration information in addition to synchrophasor measurement. However, this research provides a solution in case the location and duration are well known.

Paper [12] proposes an approach for early detection of the transient instability of the power system to initiate emergency control in time. The synchrophasor measurements are used for real-time monitoring of the system. After that, the Artificial Neural Network (ANN) is used as a classifier for predicting the transient instability status of the system with rotor angles and speeds (frequency) of the generator as inputs at different consecutive cycle lengths after fault clearing. The stability status obtained from ANN can initiate emergency control actions within a few cycles from fault clearing. 
For this work, the ability of the ANN technique to build a highly complex relationship between variables to estimate the internal behavior and response characteristic of the dynamic system, the capacity of ANN to work online with a swift response, and the benefits of its accuracy in addition to its ability to learn more cases in its live state are some motivations to use ANN in a stability prediction. Data collection from NEPLAN software for the IEEE 39-bus test system and ANN structure are presented in section 2. Then, the model simulation and ANN results are shown in section 3.

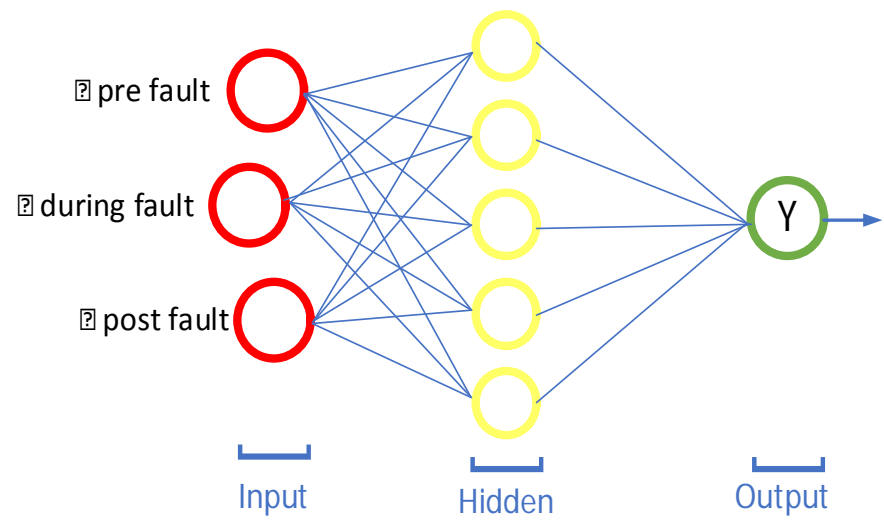

Figure 1. The general structure of ANN, the input layer (red circles), and the output layer (green) depend on the number of input and output. More than one layer may be used in the hidden layer (yellow).

\section{ARTIFICIAL NEURAL NETWORK ANN}

Artificial Neural networks (ANN) or connectionist systems are computing systems vaguely inspired by the biological neural networks that constitute animal brains [13]. Such systems" learn" to perform tasks by considering examples, generally without being programmed with task-specific rules.

ANNs have evolved into a broad family of techniques that have advanced state-of-the-art across multiple domains. The simplest types have one or more static components, including the number of units, layers, unit weights, and topology. On the other hand, dynamic types allow one or more of these to evolve via learning. These types are much more complicated but can shorten learning periods and produce better results. Some types allow/require learning to be supervised by the operator, while others operate independently. Some types operate purely in hardware, while others are purely software and run-on general-purpose computers.

The mapping between input and targetbases on the concept of neural where each neuron has its transfer function; there are many presented transfer functions in the literature. Figure 1 shows the relation between the variables in our example. For each generator, three angles are used, pre-fault, duringfault, and post-fault.
As mention above, ANN can be used to synthesize complex mapping accurately and rapidly for practical applications. Feedforward Neural Network (FFNN) is a class of ANN, generally termed Multi-Layer Perceptron (MLP), maps input data sets to a suitable output. The steps involved in detecting transient stability following significant disturbance using FFNN are presented in the next subsections.

\subsection{Data collection}

For transient stability assessment,a large number of contingencies should be simulated. The selection of the contingency depends on the operator's experience. In this study, contingencies are considered at three different locations: three-phase to ground fault (i) at the high side of all the generating buses, (ii) at the critical load buses, and (iii) at the midpoint of each transmission line. The system should be simulated for all possible contingencies with varying operating conditions to find system behavior after disturbance for all possible operating scenarios. NEPLAN generates considerable input data for three different loading levels: base case and $\pm 10 \%$ of the base case. Three-phase faults have been applied at each substation, transmission line, generator, and transformer and then removed after time. Fault clearing time is randomly chosen from 5 cycles to 12 cycles. Long-duration faults are deliberately taken to generate enough unstable cases. Thus, total operating points corresponding to 300 cases are generated where 152 are stable cases, and 148 are unstable cases.

\section{2. feature selection}

Three input features are extracted for each fault case:all generators' pre-fault, during-fault, and post fault rotor speed. Because all generators' pre-fault speeds should be the same at each scenario for the same operation point, they can be replaced by just two generators' speeds. These speeds represent the operation point, so they are changed if the operation point is changed.One generator angle is selected as a reference angle, so it is ignored for the data input. So, the ANN structure has 20 inputs and a single output.

\section{SIMULATION AND RESULT}

The proposed ANN-based approach is tested on the IEEE-39 New England test system. However,this system consists of ten generators, forty-six transmission lines, and twelve transformers. Moreover,the slack generator bus is number 39 , andthe detail generator model is modeled with exciter, turbines, stabilizers, and governors. The 3-phase fault is created at all the high sides of generator buses, load buses, and the midpoint of all the transmission lines at the base case. The data is generated offline by randomly varying load from $10 \%$ of the base case for training of FFNN. The dynamic simulation is performed for 5 seconds, and each operating condition is assigned a class label of 0 or 1 for transient unstable and transient stable state, respectively, 
based on the final values of rotor angles. The database of 300 cases is prepared to detect abnormal conditions, of which 152 are stable cases, and 148 are unstable cases. After normalization, $70 \%$ of the scenarios are taken for training the FFNN and the remaining $30 \%$ for testing and validation. All the simulations are carried out using MATLAB 2019a, and NEPLAN 5.5.5 is used to simulate IEEE 39 bus system, shown in Figure 2. Two fault duration times are applied here, short fault duration, $100 \mathrm{~ms}$, and long fault duration, $200 \mathrm{~ms}$. The effect of fault duration is shown in Figures 3 and 4. These Figures show the dynamic change in generators angles if a 3-phase fault occurs at bus 34, then the fault cleared after $100 \mathrm{~ms}$ and $200 \mathrm{~ms}$ in Figures 3 and 4, respectively. The output of the trained ANN is also presented in these figures. For the stable event, the output of the ANN is not changed (zero) and will toggle for an unstable event.

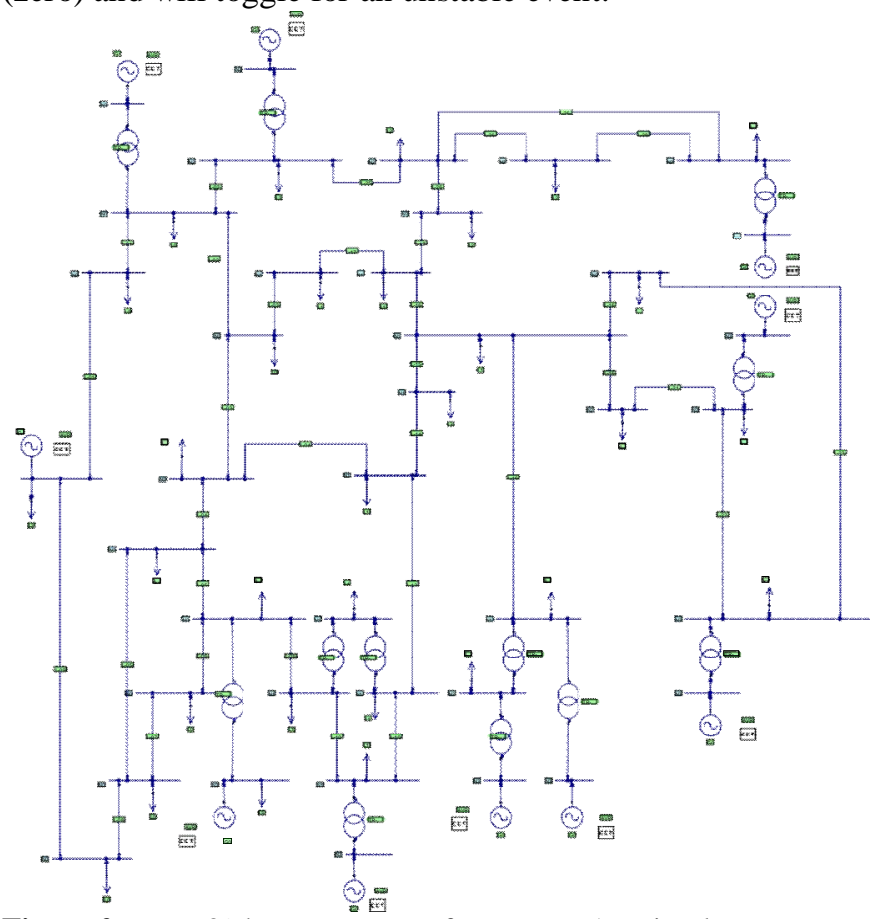

Figure 2. IEEE 39 bus test system from NEPLAN simulator consists of 10 generators and 46 lines.
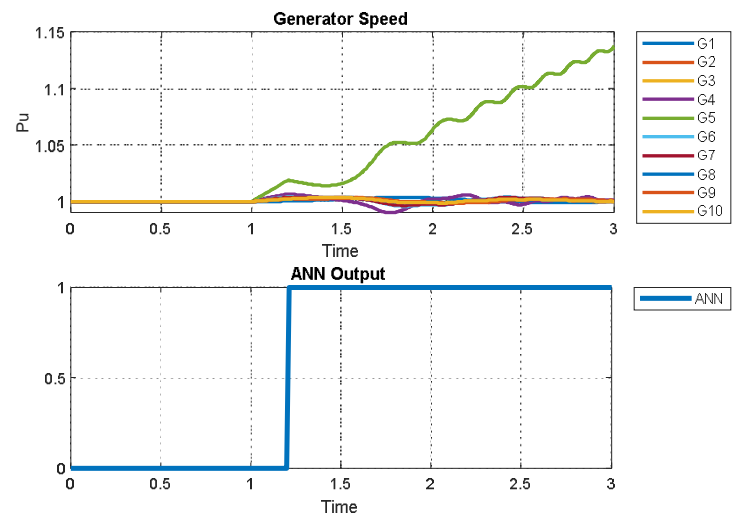

Figure 3. Generators' speeds for an unstable event, three-phase fault applied at bus number 34 for $200 \mathrm{~ms}$.
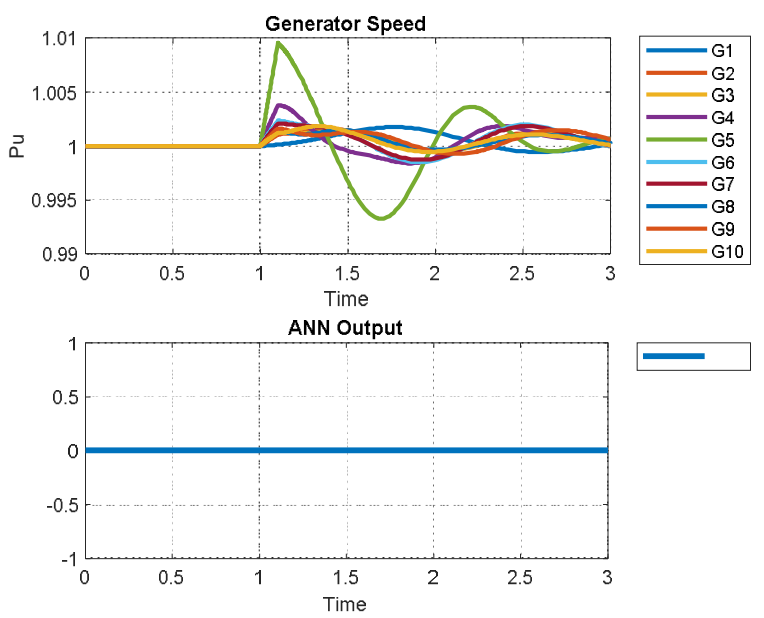

Figure 4. Generators' speeds for a stable event, three-phase fault applied at bus number 34 for $100 \mathrm{~ms}$.

For ANN training, 300 samples, 20 inputs, and one output data representing the most critical cases and extracting the best feature from the system are generated from NEPLAN to train ANN using MATLAB.

Two separate ANN models are used: the first is feed-forward ANN, and the second isa step function preceptor. All combinations of three layers with less than 25 neurons per layer are trained, and the best system is selected based on the following criteria:

Error $=\frac{\sum(\text { output }- \text { target })^{2}}{\text { numberofsample }} \times 100 \%$

The minimum error structure is a three-layer with 5,5,8 neurons per layer, respectively; the structure is shown in Figure 5. The system's error reaches $6.8 \mathrm{e}-12 \%$. After digitalize stage, this error is eliminated, which means the proposed ANN detects all stable and unstable scenarios correctly.

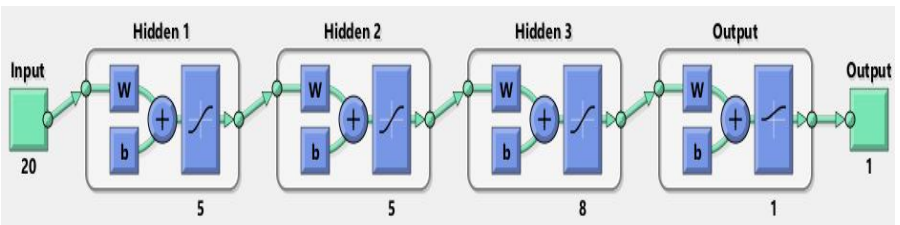

Figure 5. The first ANN structure, FFNN, consists of three hidden layers with 5,5,8 neurons per layer.

The backpropagation training algorithm conducts to determine the optimal value of each weight in the structure. The backpropagation method firstly selects random values to the weights and derive the partial derivative of the error with respect to each weight to minimize the error; therefore, this technique is highly affected by the first random values, which may (in most cases) lead the system to a local solution. for overcoming this problem, the same structure [5 58 ] is trained 2000 times. Then, the best solution defined by equation (1) is saved. 
The relative values of the weights between layersare presented in Figure 6. The red square refers to a negative value, green a positive value, and the big one represents relatively considerable weight. The absolute values of these weights are attached in Tables 1-4 in the appendix.

From Figure 6, the relative weights detect the importance of the connection; for example, inputs number 2 and 12 are the less important in this classification, but input number 13 is the most important one. So, the input can be reselection based on this character. Moreover, the location of PMUs can also be selected based on the critical input (s). The ANN structure is acceptable because the weights' values are distributed uniformly between the neurons; so, all neurons are essential to achieve the goal.

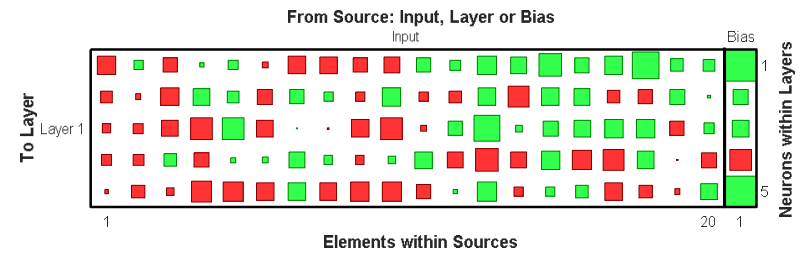

(a) Input Layer-Layer 1 relative wights

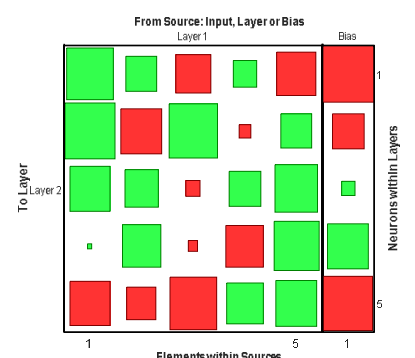

(b) Layer1-Layer 2 wights

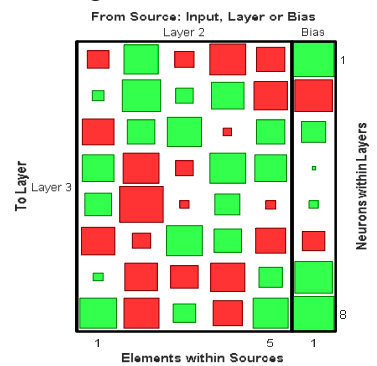

(c) Layer 2-Layer 3 wights

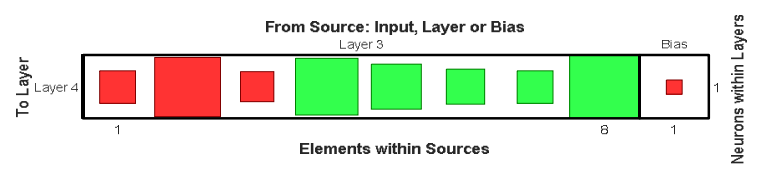

(d) Layer 3-output layer wights

Figure 6. Relative weights between input, output, and hidden layers, green refers to a positive value, and red refers to a negative value.

The performance plot is shown in Figure 7. The best validation performance (cross-Entropy of the validation set) is about $1.18 \mathrm{e}-7$ reaches at iteration 48 . The train and test performance are close to the validation. Error in the train data set has a more considerable value than validation and test data set in the training process, which means the division algorithm works correctly, and the train data set represents all data features. Finally, the confusion matrix is presented in Figure 8.

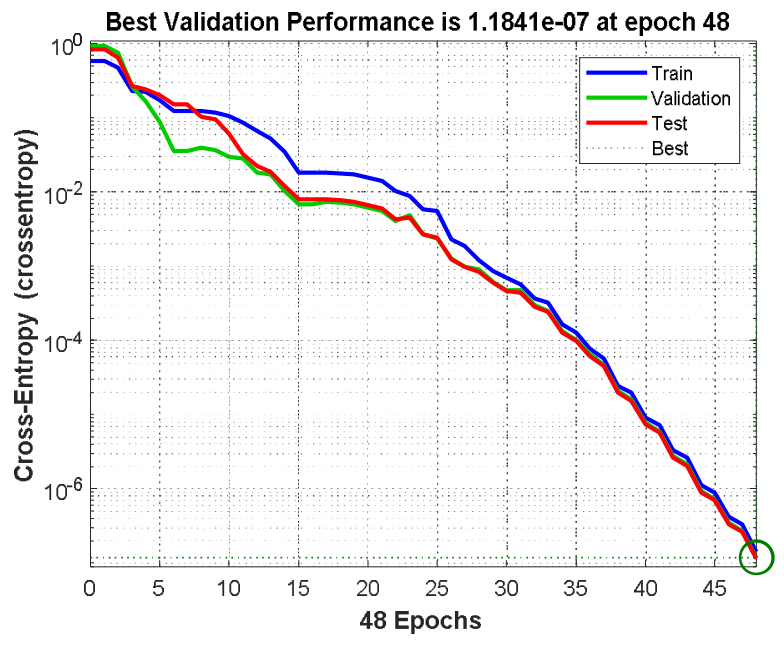

Figure 7.The performance plot of the trained ANN is divided into $70 \%, 15 \%$, and $15 \%$ for train, test, and validation, respectively.

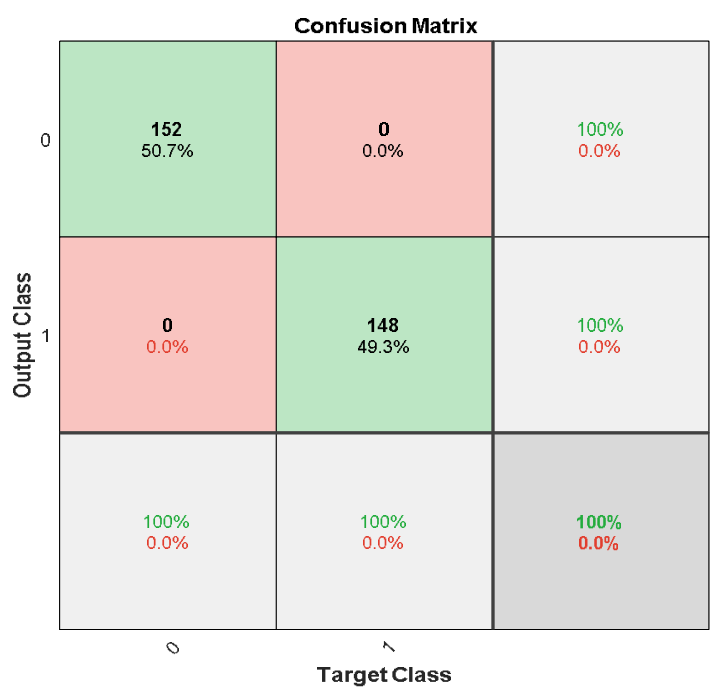

Figure 8. The confusion matrix of the trained ANN consists of 148 unstable events and 152 stable events; zero refers to a stable event and one to an unstable event.

Figure 8 shows the confusion matrix of the 148 unstable cases and 152 stable events.All these events are predicted correctly by the Proposed ANN. Green squares refer to correct prediction, and red squares refer to incorrect prediction. From the figure, four parameters can be defined as follow:

- TP: True Positive, number of correct stable event prediction to the total stable events, element $(0,0)$ from the confusion matrix.

- TN: True Negative, number of correct unstable event prediction to the total unstable events, element $(1,1)$ from the confusion matrix.

- FP: False Positive, number of incorrect stable event prediction to the total stable events, element $(0,1)$ from the confusion matrix.

- FN: False Negative, number of incorrect unstable event prediction to the total unstable events, element $(1,0)$ from the confusion matrix. 
The best classification method should give maximum TP and TN and minimum FP and FN. Figure 8 shows that the TP, $\mathrm{TN}, \mathrm{FP}$, and $\mathrm{FN}$ are $100 \%, 100 \%, 0 \%$, and $0 \%$ respectively. So this is the best classification algorithm for this problem.

\section{CONCLUSION}

An efficient method is proposed to detect the transient instability based on rotor angle speed obtained through synchrophasor measurements across the system in real-time. The critical feature of the method is that the developed FFNN output depends upon the post-fault scenarios and is independent of fault location and type of fault. The proposed ANN used two cascade ANN, one FFNN and the second is step perceptron. The benefit of usingtwo-level makes a rest pointinsidethe ANN structure, which helps the learning algorithm fit the globularity more accurately. The output of the ANN can be utilized for initiating emergencycontrol actions. The proposed method is suitable for detecting transient instability and early initiation of the Remedial Action Scheme, considering the time requirements for implementing the emergency actions.

\section{APPENDIX}

Table 1. Input hidden layers weights

\begin{tabular}{|l|l|l|l|l|l|}
\hline $\mathbf{I} / \mathbf{H 1}$ & $\mathbf{1}$ & $\mathbf{2}$ & $\mathbf{3}$ & $\mathbf{4}$ & $\mathbf{5}$ \\
\hline $\mathbf{1}$ & -0.513 & -0.216 & -0.113 & -0.172 & -0.018 \\
\hline $\mathbf{2}$ & 0.125 & -0.048 & -0.148 & -0.176 & -0.259 \\
\hline $\mathbf{3}$ & -0.3 & -0.481 & -0.402 & 0.234 & -0.084 \\
\hline $\mathbf{4}$ & 0.024 & 0.422 & -0.719 & -0.326 & -0.608 \\
\hline $\mathbf{5}$ & 0.138 & 0.206 & 0.716 & 0.038 & -0.594 \\
\hline $\mathbf{6}$ & -0.056 & -0.348 & -0.413 & 0.05 & -0.471 \\
\hline $\mathbf{7}$ & -0.467 & 0.315 & 0 & 0.378 & 0.448 \\
\hline $\mathbf{8}$ & -0.437 & 0.132 & -0.004 & 0.112 & -0.431 \\
\hline $\mathbf{9}$ & -0.319 & -0.118 & -0.47 & -0.109 & -0.513 \\
\hline $\mathbf{1 0}$ & -0.371 & 0.509 & -0.709 & 0.092 & -0.543 \\
\hline $\mathbf{1 1}$ & 0.298 & -0.116 & -0.051 & 0.426 & -0.322 \\
\hline $\mathbf{1 2}$ & 0.161 & -0.213 & 0.298 & -0.362 & 0.021 \\
\hline $\mathbf{1 3}$ & 0.531 & 0.445 & 1.021 & -0.733 & 0.531 \\
\hline $\mathbf{1 4}$ & 0.401 & -0.663 & 0.068 & -0.359 & -0.107 \\
\hline $\mathbf{1 5}$ & 0.776 & 0.483 & 0.344 & 0.515 & 0.111 \\
\hline $\mathbf{1 6}$ & 0.327 & 0.406 & 0.42 & -0.522 & 0.245 \\
\hline $\mathbf{1 7}$ & 0.49 & -0.246 & 0.518 & -0.647 & -0.477 \\
\hline $\mathbf{1 8}$ & 1.054 & -0.311 & 0.521 & 0.428 & -0.261 \\
\hline $\mathbf{1 9}$ & 0.227 & 0.357 & -0.314 & -0.003 & -0.043 \\
\hline $\mathbf{2 0}$ & 0.221 & 0.013 & 0.163 & -0.342 & 0.406 \\
\hline
\end{tabular}

Table 2. Hidden layer 1 to hidden layer 2 weights

\begin{tabular}{|l|l|l|l|l|l|}
\hline $\mathbf{H 1} / \mathbf{H} 2$ & $\mathbf{1}$ & $\mathbf{2}$ & $\mathbf{3}$ & $\mathbf{4}$ & $\mathbf{5}$ \\
\hline $\mathbf{1}$ & 1.472 & 1.716 & 1.087 & 0.013 & -1.061 \\
\hline $\mathbf{2}$ & 0.655 & -1.111 & 0.766 & 0.995 & -0.58 \\
\hline $\mathbf{3}$ & -0.827 & 1.601 & -0.133 & -0.059 & -1.485 \\
\hline $\mathbf{4}$ & 0.383 & -0.088 & 0.66 & -0.96 & 0.92 \\
\hline $\mathbf{5}$ & -1.033 & 0.645 & 1.23 & 1.34 & 1.118 \\
\hline
\end{tabular}

Table 3. Hidden layer 1 to hidden layer 2 weights

\begin{tabular}{|l|l|l|l|l|l|}
\hline $\mathrm{O} / \mathrm{H} 3$ & $\mathbf{1}$ & $\mathbf{2}$ & $\mathbf{3}$ & $\mathbf{4}$ & $\mathbf{5}$ \\
\hline 1 & -0.54 & 1.36 & -0.43 & -1.51 & -0.97 \\
\hline 2 & 0.15 & 1.69 & 0.36 & 1.2 & -1.31 \\
\hline 3 & -1.11 & 0.9 & 1.36 & -0.08 & 0.97 \\
\hline 4 & 1.11 & -1.49 & -0.37 & 1.52 & 1.13 \\
\hline 5 & 0.79 & -2.16 & -0.09 & 0.74 & -0.11 \\
\hline 6 & -1.26 & -0.39 & 1.47 & 0.86 & -1 \\
\hline 7 & 0.1 & -1.21 & -0.87 & -1.35 & 0.64 \\
\hline 8 & 1.5 & -1.46 & 0.57 & -1.01 & 1.46 \\
\hline
\end{tabular}

Table 4. Hidden layer 3 to output layer weights

\begin{tabular}{|l|l|l|l|l|l|l|l|l|}
\hline $\mathbf{H 3}$ & $\mathbf{1}$ & $\mathbf{2}$ & $\mathbf{3}$ & $\mathbf{4}$ & $\mathbf{5}$ & $\mathbf{6}$ & $\mathbf{7}$ & $\mathbf{8}$ \\
\hline $\mathbf{O}$ & -1.2 & -3.9 & -1 & 3.54 & 2.25 & 1.35 & 1.15 & 4.36 \\
\hline
\end{tabular}

\section{REFERENCES}

[1] Odienat, Abdullah, et al. "Low Frequency Oscillation Analysis for Dynamic Performance of Power Systems." 2021 12th International Renewable Engineering Conference (IREC). IEEE, 2021.

[2] M. M. Almomani, A. Odienat, S. F. Al-Gharaibeh and K. Alawasa, "The Impact of Wind Generation on Low Frequency Oscillation in Power Systems," 2021 IEEE PES/IAS PowerAfrica, 2021, pp. 1-5, doi: 10.1109/PowerAfrica52236.2021.9543283.

[3] Almomani, Mohammad M., and Seba F. Algharaibeh. "Modelling and Testing of a Numerical Pilot Distance Relay for Compensated Transmission Lines." International Journal of Scientific Research and Engineering Development 3.6 (2020): 776-786.

[4] Al-Momani, Mohammad M., Asmaa SM Hatmi, and Seba F. Al-Gharaibeh. "European Journal of Electrical Engineering." European Journal of Electrical Engineering 23.3 (2021): 197-205.

[5] Al-Odienat, A. I., et al. "Connectivity Matrix Algorithm: A New Optimal Phasor Measurement Unit Placement Algorithm." IOP Conference Series: Earth and Environmental Science. Vol. 551. No. 1. IOP Publishing, 2020.

[6] Almomani, Mohammad, Ali S. Al-Dmour, and SebaAlgharaibeh. "Application of Artificial Intelligence Techniques for Modeling and Simulation of Photovoltaic Arrays."

[7] M. Mahdi and V. I. Genc, "Artificial neural network based algorithm for early prediction of transient stability using wide area measurements," in 2017 5th International Istanbul Smart Grid and Cities Congress and Fair (ICSG). IEEE, 2017, pp. 17-21.

[8] S. Suliman and T. A. Rahman, "Artificial immune system based machine learning for voltage stability prediction in power system," in 2010 4th International Power Engineering and Optimization Conference (PEOCO). IEEE, 2010, pp. 53-58.

[9] Multi-Terminal, H. V. D. C. "Mohammad Al-Momani, Seba Al-Gharaibeh1, Hussein. D. Al-Majali2 Mutah University." 
[10] V. Malbasa, C. Zheng, P.-C. Chen, T. Popovic, and M. Kezunovic, "Voltage stability prediction using active machine learning," IEEE Transactions on Smart Grid, vol. 8, no. 6, pp. 3117-3124, 2017.

[11] M. Mahdi and V. I. Genc, "Artificial neural network based algorithm for early prediction of transient stability using wide area measurements," in 2017 5th International Istanbul Smart Grid and Cities Congress and Fair (ICSG). IEEE, 2017, pp. 17-21.

[12] S. A. Siddiqui, K. Verma, K. Niazi, and M. Fozdar, "Artificial neural network based early detection of real-time transient instability for initiation of emergency control through wide-area synchrophasor measurements," in 2015 International Conference on Computer, Communication and Control (IC4). IEEE, 2015, pp. 1-6.

[13] YADDANAPUDI, KIRAN, and PANNALA KRISHNA MURTHY. "PERFORMANCE ANALYSIS OF FUZZY LOGIC CONTROLLED HYBRID ACTIVE DC FILTER (HADF) FOR 12-PULSE HVDC CONVERTER." International Journal 9.2 (2021). 